Highlight Article: 2002 ACMT/Orphan Medical Award for Antidotal Research Winmer

\title{
Vasopressin Treatment of Verapamil Toxicity in the Porcine Model
}

\author{
J. Dave Barry, MD a, Dave Durkovich, D.O.b, Lee Cantrell, PharmD ${ }^{\mathrm{c}}$, William Richardson, MD ${ }^{\mathrm{d}}$, \\ Tri Tong, MDe, Steve Offerman, MD f , Richard F. Clark, MDg, David A. Tanen, MD h, \\ Saralyn Williams, MDg
}

\author{
a Department of Emergency Medicine, Brooke Army Medical Center, Fort Sam Houston, TX \\ b Department of Emergency Medicine, Naval Hospital, Sigonella, Italy \\ c California Poison Control System, San Diego Division, San Diego, CA \\ d Department of Emergency Medicine, Palmetto Richland Memorial Hospital, Palmetto Poison Center, Columbia, SC \\ e Department of Emergency Medicine, South Coast Medical Center, Laguna Beach, CA \\ f Department of Emergency Medicine, UC Davis School of Medicine, Sacramento, CA \\ g UCSD Division of Medical Toxicology, San Diego Division of the California Poison Control System, University of California, \\ San Diego (UCSD) Medical Center, San Diego, CA \\ h Department of Emergency Medicine, Naval Medical Center San Diego, San Diego, CA
}

\section{ABSTRACT}

Introduction: Vasopressin is a novel vasopressor agent used for intractable hypotension. There is little published data available on its use in the poisoned patient. We performed a randomized, controlled, blinded trial in a porcine model to study the effects of vasopressin infusion on mean arterial pressure after verapamil poisoning.

Methods: Eighteen anesthetized monitored swine received a verapamil infusion of $1 \mathrm{mg} / \mathrm{kg} / \mathrm{hr}$ until the mean arterial pressure (MAP) had decreased to $70 \%$ of baseline. At this time, a continuous infusion of either vasopressin $(0.01 \mathrm{U} / \mathrm{kg} / \mathrm{min}) \mathrm{or}$ an equal volume of normal saline was initiated. The swine were monitored for 60 minutes after initiation of the study infusion. The primary outcome was MAP.

Results: There was no statistically significant difference between the two groups in MAP, cardiac output or systemic vascular resistance. One half (four of eight) of the animals in the vasopressin group died, compared with $20 \%$ (two of ten) of those in the saline group.

Conclusions: Vasopressin infusion decreased the survival of verapamil-poisoned swine when compared to those treated with saline alone in this experimental model.

Keywords: vasopressins, verapamil, drug toxicity

Notes: This research was generously supported by the ACMT research award: a \$10,000 grant funded equally by the American College of Medical Toxicology and Orphan Medical. This research was orally presented at the North American Congress of Clinical Toxicology (NACCT) Annual Conference, Sept 03. An abstract was published in J Toxicol Clin Toxicol 2003;41(5):694. We would like to recognize Dr Robert Riffenberg, Dr John Ward (for stastical support) and Dr Steven Curry (for laboratory support). We bestow them a very special thanks for their invaluable contributions. The views expressed in this article are those of the authors and do not necessarily reflect the official policy or position of the U.S. Army, U.S. Navy, Department of Defense, nor the U.S. Government.

Corresponding Author: Maj. J. Dave Barry, MD, Department of Emergency Medicine, Brooke Army Medical Center, 3851 Roger Brooke Drive, Fort Sam Houston, TX 78234. Email: JDave.Barry@us.army.mil 


\section{INTRODUCTION}

Calcium Channel Blockers (CCBs) are among the most widely prescribed antihypertensives in use today. In 1989, verapamil, diltiazem and nifedipine were among the top 20 prescription drugs dispensed by pharmacies [1] and their use has continued to increase since that time. In 2003, the American Association of Poison Control Centers (AAPCC) reported over 9600 exposures to these agents, including nearly 1500 characterized by moderate to major toxicity, and 57 deaths [2,3]. This report described cardiovascular drugs as among the five most lethal classes of pharmaceutical agents in overdose settings. Within the category of cardiovascular agents, CCBs continue to be responsible for the majority of deaths. CCBs block L-type voltage-gated calcium channels, impeding the influx of calcium that is critical for contraction in cardiac and vascular smooth muscles. Therapeutic doses slow cardiac conduction, decrease contractility and lead to peripheral vasodilation. In overdose, intractable hypotension and bradydysrhythmias are common.

Historically, verapamil has been the CCB associated with the most deaths [4]. Many patients suffering from a mild overdose of CCBs may respond to supportive therapy and intravenous calcium, however, patients with severe poisoning can be refractory to all conventional therapies.

CCB poisoning is especially concerning since no consistently effective treatment for massive overdose of these agents has been found. Case reports document the inability of conventional interventions, alone or in combination, to prevent death following severe cases of verapamil poisoning $[5,6]$. CCB toxicity results in treatment difficulties similar to those of cardiac arrest and various hypotensive shock states: a depressed response to volume replacement and standard vasopressor therapy. Vasopressin (AVP) has shown significant benefit in the treatment of these states [7-17] and we hypothesized that it may also benefit severe CCB toxicity.

This study was undertaken to evaluate the efficacy of AVP as a therapy in verapamil overdose. The rationale for using vasopressin is based on its ability to increase intracellular calcium and data showing benefit in other refractory hypotensive states. We hypothesized that vasopressin infusion would lead to a $50 \%$ increase in mean arterial blood pressure (MAP) when compared to saline infusion in verapamil poisoned pigs.

\section{MATERIALS AND METHODS}

\section{Study design}

This was a blinded, prospective randomized controlled animal experiment evaluating the effectiveness of vasopressin in swine poisoned with verapamil using MAP as the main outcome parameter. The study hospital's animal use committee approved the study protocol. The care and handling of animals was in accord with National Institutes of Health guidelines for ethical animal research.

We considered an increase in MAP of $50 \%$ in the treatment group to be clinically meaningful. With a two-tailed alpha of .05, we determined that 9 animals in each group would have a power of 0.8 to detect a $50 \%$ increase in MAP in the experimental group.

\section{Data collection and processing}

Twenty-four immature male swine weighing between $28-48 \mathrm{~kg}$ were obtained. Six animals were used to formalize dosing regimens and 18 animals were used for the formal study.

Dosing studies were performed in the same manner as described below, but the severity of poisoning (based on \% of baseline MAP) was varied to find an appropriate level of poisoning to allow animals to survive the study period. Initial intentions were to poison animals to $45 \%$ of baseline MAP, but this resulted in rapid death of the animal. Poisoning (based on \% of baseline MAP) was adjusted to a point where it was felt animals would consistently survive poisoning. Six animals were utilized to formalize the poisoning regimen.

Each animal was acclimatized per study hospital laboratory protocol, prior to the study. Animals were maintained on a standard diet, until the night preceding the study. Animals were then fasted overnight (12 hours) except for water ad lib. Animals were weighed the morning of the experiment.

Initial sedation was produced with Telazol (tiletamine and zolazepam) 54 milligrams(mg)/kilogram(kg) by intramuscular (IM) injection. Animals were then intubated and ventilated with a mixture of $60 \%$ oxygen, $40 \%$ nitrogen and isoflurane. The anesthetic agent was titrated to maintain animal comfort. Mechanical ventilation was adjusted to keep arterial PCO2 at 40 millimeters of Mercury ( $\mathrm{mm} \mathrm{Hg}$ ) during a 30-minute (min) stabilization period.

Electrocardiogram (ECG) electrodes were placed for continuous monitoring of heart rate and rhythm. An intravenous (IV) catheter was placed in an ear vein and each animal received 30 milliliters $(\mathrm{mL}) / \mathrm{kg}$ normal saline during the stabilization period.

Arterial and central venous access was accomplished by cutdown to isolate the internal jugular vein and carotid artery. The vein was cannulated with a thermo dilution cardiac output Swan Ganz catheter and advanced into the pulmonary artery. Cardiac output was measured utilizing a thermo dilution method and central venous pressure was continuously monitored. A 3.5-inch catheter was placed in the carotid artery and attached to a transducer for continuous monitoring of arterial blood pressure. Blood sampling was obtained through the arterial catheter.

After the 30-min. stabilization period to ensure stable blood pressure, heart rate and cardiac output, vital sign and laboratory values were documented as baseline values.

Baseline laboratory values included arterial blood gases, plasma potassium, plasma verapamil concentration, blood glucose and blood ionized calcium concentration. Blood gases, plasma potassium, blood glucose and blood ionized calcium were obtained using an IRMA SL Blood Analysis System Series 2000 (Diametrics Medical, Inc.) that was located within the laboratory and provided rapid bedside results. Blood for plasma verapamil concentrations was centrifuged after acquisition to obtain the serum component. Serum was then frozen and sent 
to Quest Diagnostics-Nichols Institute (San Juan Capistrano, CA) for quantification.

Poisoning was performed with verapamil infusion. Intravenous preparations of verapamil hydrochloride $(0.5 \mathrm{mg} / \mathrm{mL}$ concentration) were prepared by the study hospital's pharmacy department and provided to the research team at the beginning of each day. These verapamil infusions were initiated at $1 \mathrm{mg} /$ $\mathrm{kg} /$ hour(hr) after obtaining baseline data values and labs. Verapamil infusion was discontinued after MAP had dropped to $70 \%$ of the baseline value. This time was recorded as Time 0 .

At time zero, each animal received a study drug. Study drug was randomized and prepared by the study hospital's pharmacy department. All investigators were blinded as to which agent the animals received until the conclusion of the experiments. Animals received either $0.01 \mathrm{Units}(\mathrm{U}) / \mathrm{kg} / \mathrm{min}$ vasopressin IV for $60 \mathrm{~min}$. or an equal volume of infusion of normal saline over 60 min. Dosing experiments using vasopressin in the immature swine have been performed and this information was used as a basis for choosing the vasopressin dose used in our protocol [18]. Mean arterial blood pressure was recorded every five min. for 60 min. Heart rate, cardiac output, arterial blood gas measurments, and serum potassium and glucose, and blood ionized calcium were also measured every five minutes until time 60 minutes. Plasma verapamil concentration and plasma vasopressin concentration were measured at $\mathrm{T}_{0}, 30$ minutes and 60 minutes. Blood for plasma vasopressin concentration was prepared in the same way as that for plasma verapamil concentration. Quantification of vasopressin was also performed by Quest Diagnostics-Nichols Institute (San Juan Capistrano, CA).

Death was defined by asystole or profound sustained bradycardia with a near undetectable MAP. Surviving animals were euthanized with IV buthanesia as per standard laboratory protocol at the completion of the experiment.

\section{Outcome measures/primary data analysis/ data presentation}

Mean arterial pressure was the primary outcome variable. Mean values for MAP at 5-min. intervals were compared between groups using repeated measures analysis of variance.

Secondary outcome variables include survival, plasma verapamil concentration, plasma vasopressin concentration, heart rate and cardiac output. These values were compared between groups using repeated measures multivariate analysis of variance (MANOVA).

Death rates were not included in the original study design. Differences in deaths between the 2 groups were compared using Fisher's exact test.

\section{RESULTS}

Average animal weights were $31.8 \pm 8.5 \mathrm{~kg}$ and $32.2 \pm 6.8 \mathrm{~kg}$ in the vasopressin and saline groups respectively.

Four of 8 animals in the vasopressin group died, compared to 2 of 10 in the control group $(p=0.32)$. Death appeared to be primarily due to hypotension and substantially low cardiac output. Profound bradycardia did not occur. There was little difference in heart rate between the two groups (Figure 1).

There was no significant difference in mean arterial pressure between the two groups ( $\mathrm{p}=0.70$ ) (Figure 2). There was no significant difference in cardiac output (CO) between the 2 groups $(\mathrm{p}=0.38)$ (Figure 3). The raw data from the vasopressin group appeared to show a tendency toward increased systemic venous resistance when compared to controls, but did not meet statistical significance $(\mathrm{p}=0.07)$ (Figure 4).

There were no statistical differences in vasopressin levels between the groups at baseline and before treatment $\left(\mathrm{T}_{0}\right)$. Vasopressin treated animals showed markedly increased, but widely variable, vasopressin levels during treatment. (Figure 5) As expected, verapamil levels gradually decreased with time after poisoning. Differences in verapamil levels between groups were not statistically significant. (Figure 6)

\section{DISCUSSION}

\section{Limitations}

Our original plan for poisoning was based on previously published articles [19]. We were unable to reproduce poisoning to the same degree without our animals quickly dying. A number of factors could have contributed to our inability to reproduce these previous poisoning techniques, including differences in laboratory technique, differences in animal size, or differences in animal species, family, or age. Faced with this difficulty, we utilized 6 animals to standardize a new poisoning regimen. Our new regimen resulted in less severe verapamil poisoning (decrease in MAP to $70 \%$ of baseline vs. $50 \%$ of baseline) than previously published studies, but still resulted in an unexpectedly high death rate.

Death was not used as a primary outcome parameter as we expected the majority of animals to survive poisoning. Since our study design did not include death as an outcome parameter, differences in death rates may be misleading and could be related to other limitations in the study or due to chance alone.

Statistical analysis excluded data from deceased animals. This data set was chosen since it biased the results in favor of the vasopressin group providing the best possibility of revealing a potential benefit. As the results demonstrate, even with the data biased in favor of vasopressin, there was no benefit to its use.

Including the data from deceased animals until the time of death dramatically increased the standard deviation of each data point since dying animals in both groups occasionally survived for prolonged periods in agonal states. This made interpretation difficult because results varied with times of death instead of differences in study drug.

Our study was designed to detect a $50 \%$ increase in MAP in the treatment group. Proper laboratory technique would have required us to revise our sample size calculations after changing the method of poisoning. This was not done, causing our study to be underpowered to detect smaller changes between the two treatment groups. 


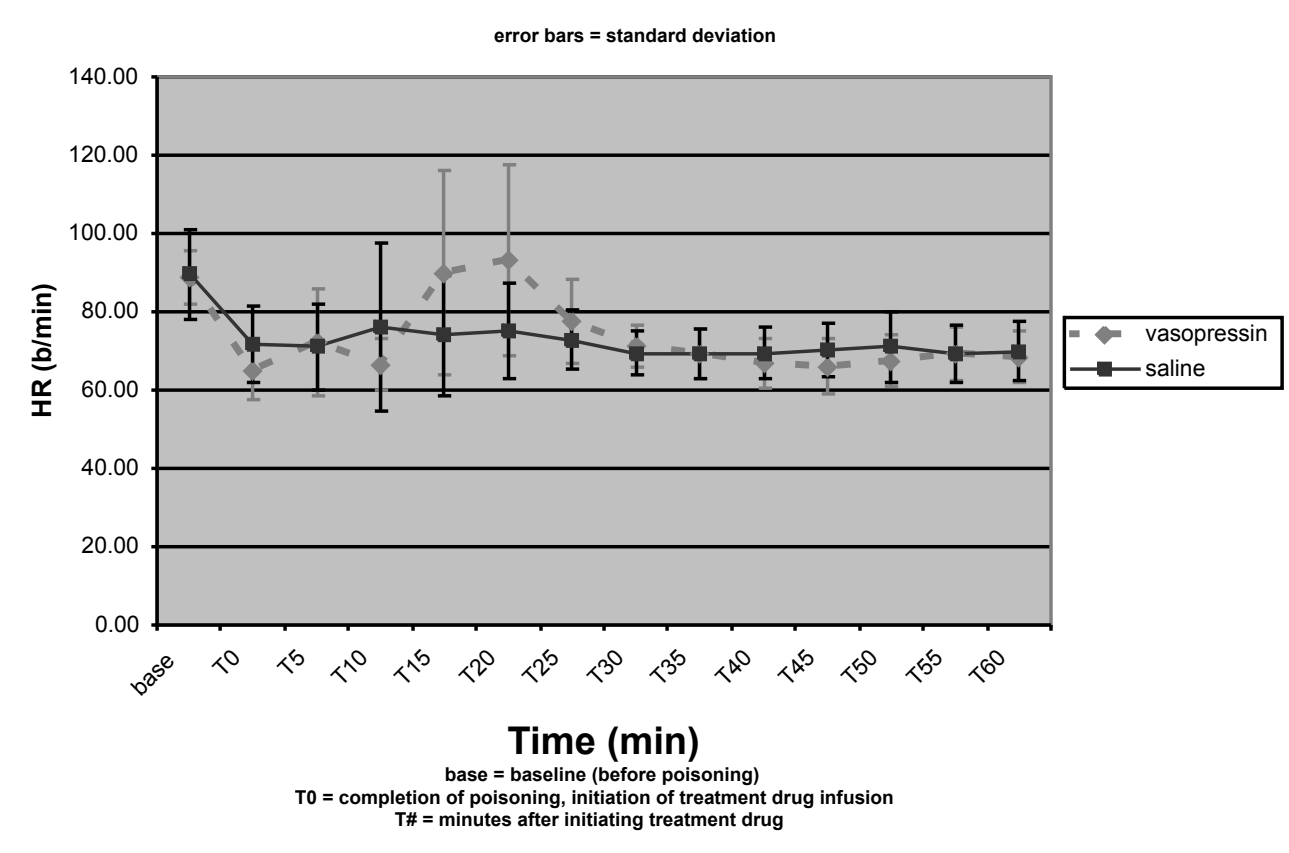

Figure 1. Average Heart Rate (HR) of Vasopressin-treated vs. Control Animals over Time in a Procine Model of Verapamil Poisoning.

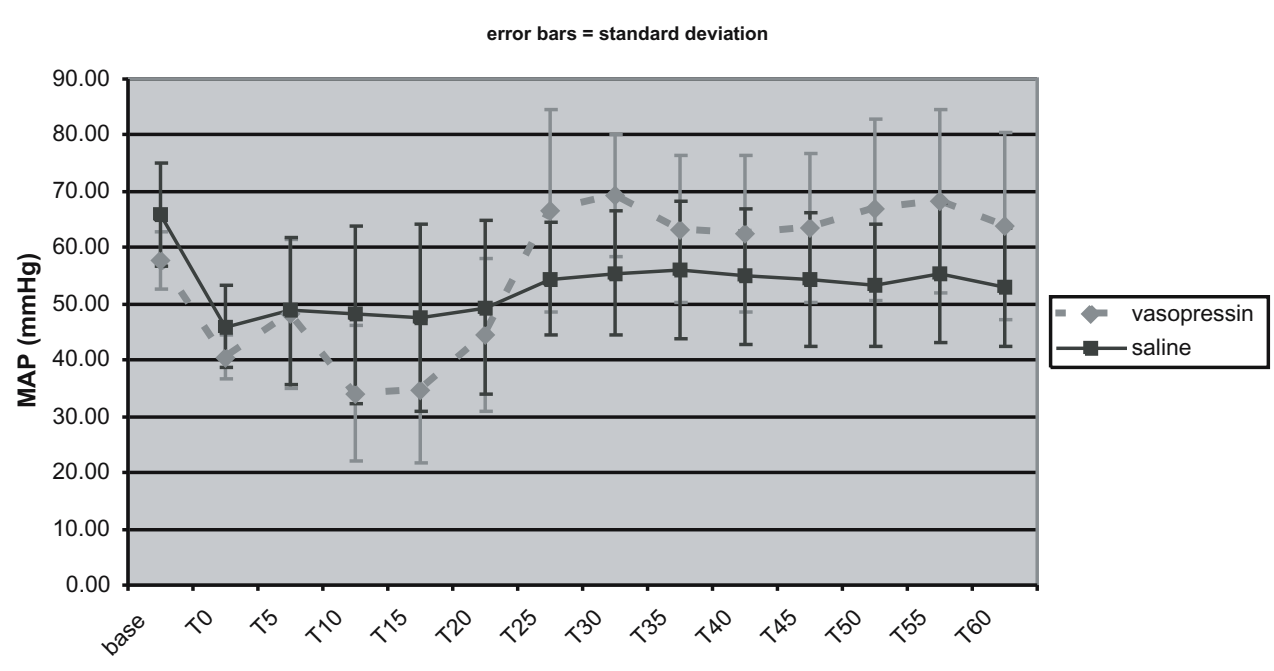

Time (min)

base $=$ baseline (before poisoning)

T0 = completion of poisoning, initiation of treatment drug infusion

$\mathrm{T \#}=$ minutes after initiating treatment drug

Figure 2. Average Mean Arterial Pressure (MAP) of Vasopressin-treated vs. Control Animals over Time in a Procine Model of Verapamil Poisoning. 
error bars = standard deviation

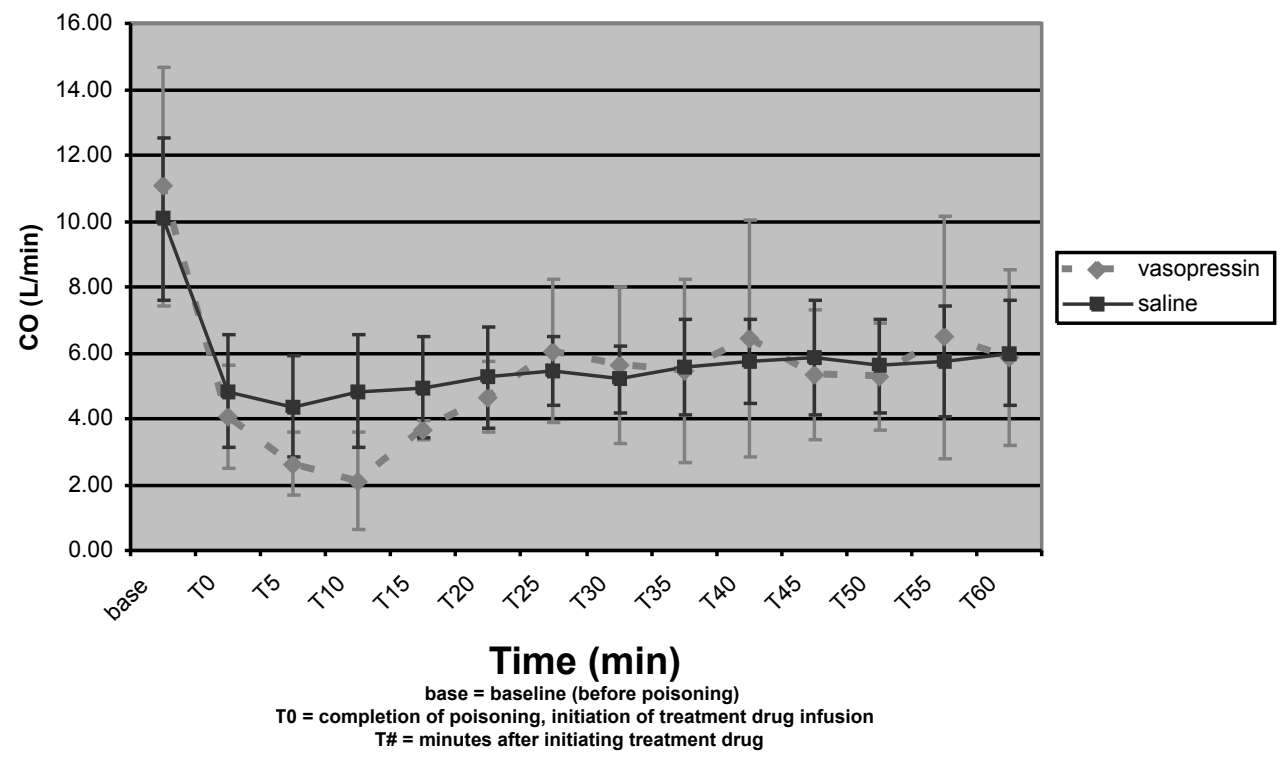

Figure 3. Average Cardiac Output (CO) of Vasopressin-treated vs. Control Animals over Time in a Procine Model of Verapamil Poisoning.

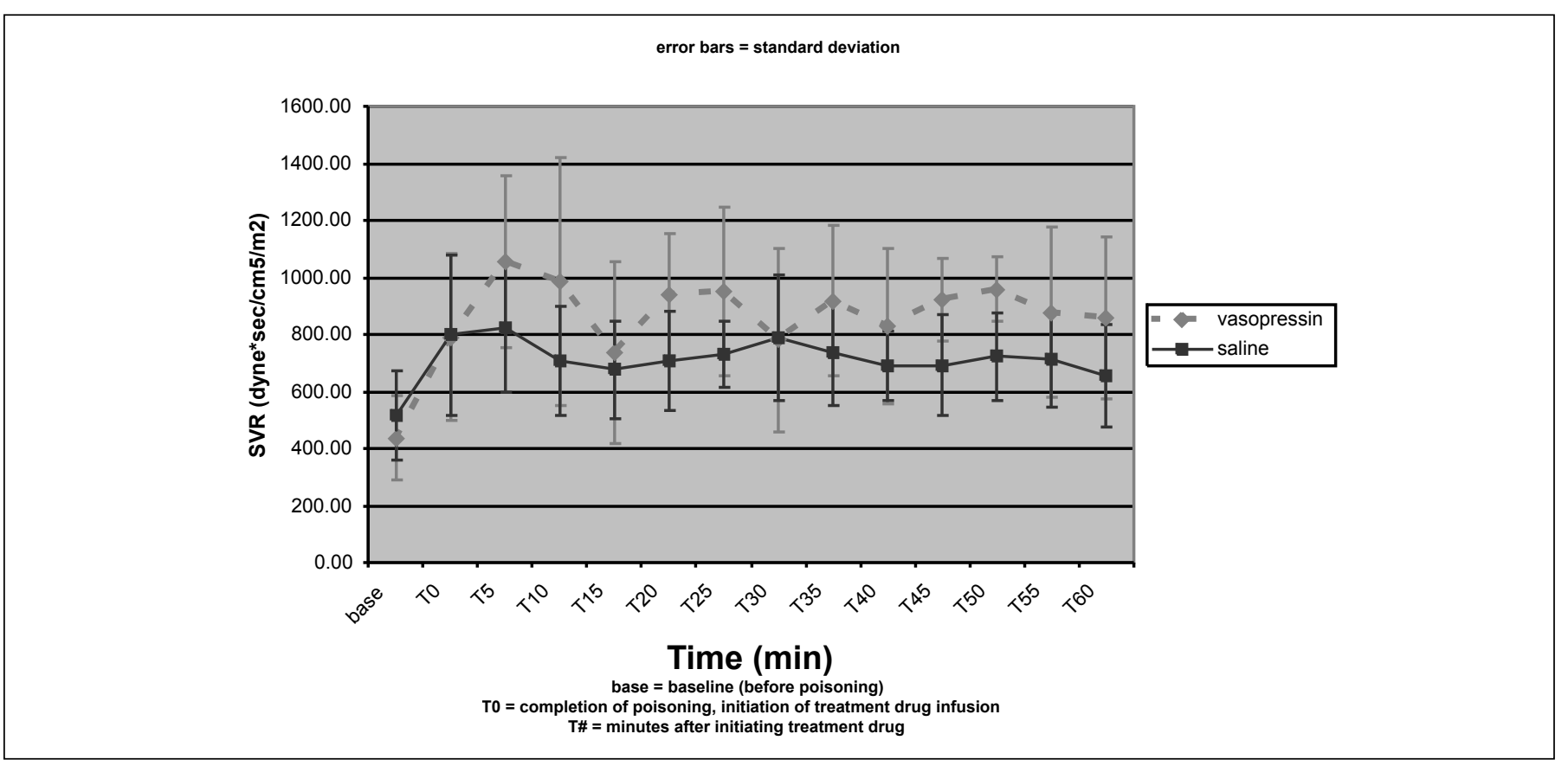

Figure 4. Average Systemic Vascular Resistance (SVR) of Vasopressin-treated vs. Control Animals over Time in a Procine Model of Verapamil Poisoning. 


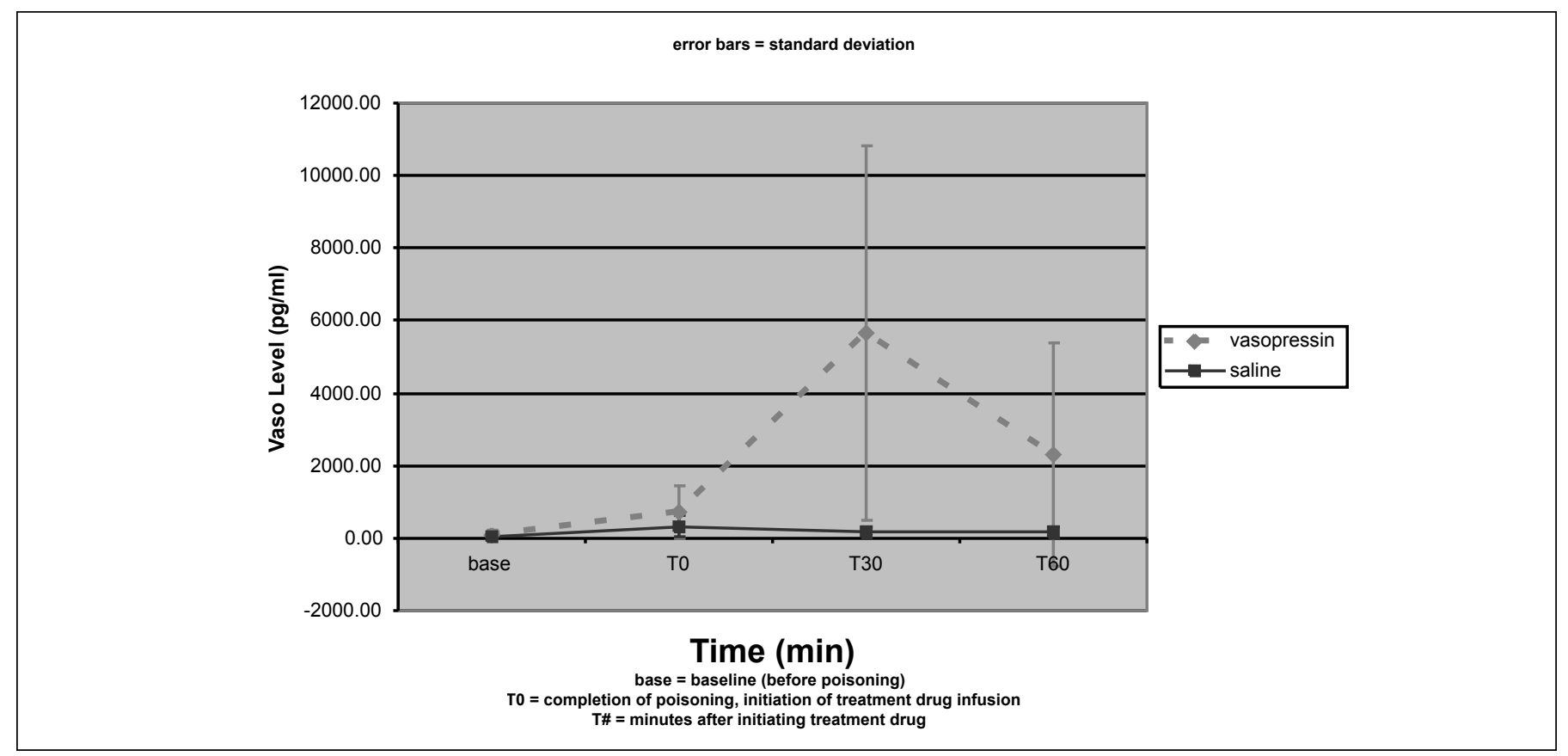

Figure 5. Average Vasopressin Levels of Vasopressin-treated vs. Control Animals over Time in a Procine Model of Verapamil Poisoning.

The primary variable of MAP was lower at baseline and at T0 in the vasopressin group. This difference could suggest a selection bias and may have contributed to the increased death rate in the vasopressin group.

As with any animal model, the results may not directly correlate with potential clinical effects seen in humans. Intravenous verapamil poisoning may not adequately mimic oral ingestion due to the wide variability of absorption and changing saturation/metabolic kinetics.

We chose a swine model because the swine cardiovascular system is considered closely analogous to that of human beings. Swine have been used in previous investigations of verapamil

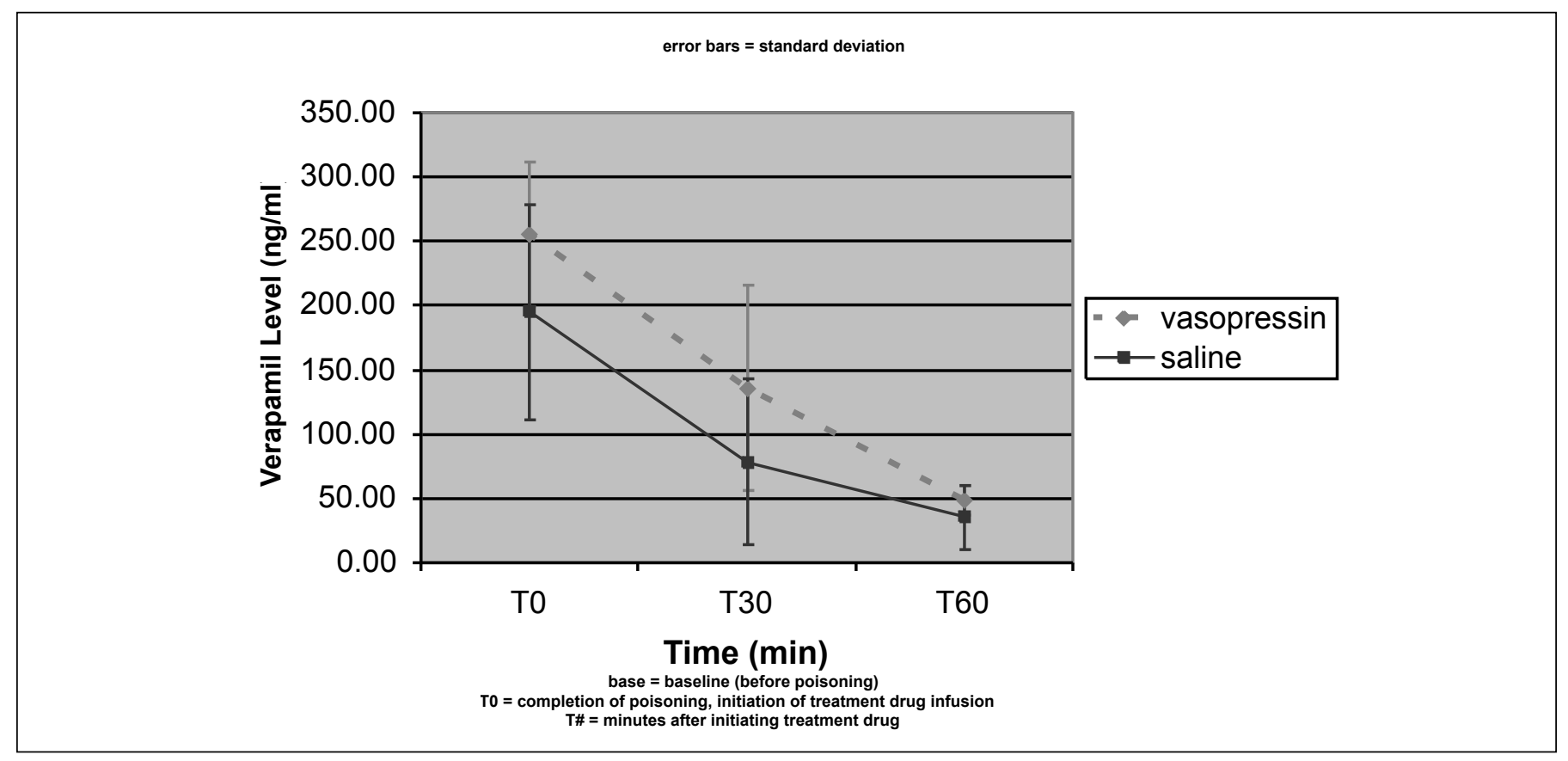

Figure 6. Average Verapamil Levels of Vasopressin-treated vs. Control Animals over Time in a Procine Model of Verapamil Poisoning. 
toxicity $[19,20]$ and in experimental models of AVP therapy [2,21-25]. Vasopressin receptors in pigs (lysine vasopressin) however, are different than those in humans (arginine vasopressin) and may result in different hemodynamic responses to exogenously administered AVP. Arginine vasopressin levels may not be accurate predictors of total vasopressin levels in the swine model.

\section{General Discussion/Magnitude of Results}

Vasopressin is a hormone released from the posterior pituitary when water deprivation or other factors lead to increased plasma osmolality, hypovolemia or hypotension. The primary action of AVP is on the cells of the renal collecting duct. For this reason, AVP is commonly referred to as Antidiuretic Hormone. AVP, as its name suggests, is also a potent vasoconstrictor. AVP's vasopressor properties have recently been shown to be beneficial in the setting of cardiac arrest and various shock states [7-17].

The vasoconstrictive effects of AVP are mediated by secondary messengers that ultimately increase intracellular calcium. The binding of AVP to its receptor leads to a number of cellular events, the most important being the hydrolysis of phosphatidylinositol1,4,5-biphosphate to inositol-1,4,5-triphosphate (IP3). IP3 binds to receptors on calcium channels located on intracellular calcium stores, leading to increased intracellular calcium concentrations. Although the mechanism is unclear, AVP receptors also cause an increased calcium influx from the extracellular compartment [26]. Adrenergic alpha ${ }_{1 \mathrm{~A}}$ receptors employ the same secondary messengers to produce increased intracellular calcium concentrations.

In the smooth muscle cells of blood vessels, increased intracellular concentrations of calcium leads to vasoconstriction. Based on this proposed mechanism, AVP would primarily affect SVR as AVP is not known to have effects on CO or contractility. The cardiac effects of verapamil, most specifically its negative effects on contractility and CO were not significantly improved with vasopressin treatment in our experiment. Our results showed no benefit in the use of vasopressin therapy for verapamil poisoning, and in addition, showed a trend toward increased mortality.

Traditional ionotropic agents and vasopressors (epinephrine, norepinephrine, etc) have been shown to have diminished action in the setting of vasodilatory shock states $[7,13-15]$. Inappropriately low plasma AVP concentrations for the degree of hypotension have been documented in these settings [13-15]. Low dose AVP infusions have resulted in significant increases in arterial blood pressure in these life-threatening situations [13-16]. Inappropriately low plasma AVP concentrations have been hypothesized as an explanation of the benefit of vasopressin in vasodilatory shock states [13-15]. Our method of verapamil poisoning resulted in no evidence of vasopressin depletion. A similar dog study evaluating vasopressin levels during verapamil poisoning also found no depletion [27]. Although our study revealed no benefit of vasopressin, different methods of creating
CCB poisoning or prolonged poisoning times that do lead to a relative depletion of vasopressin could produce different results.

Vasopressin may also potentiate the vasoconstrictor effects of traditional ionotropic agents such as norepinephrine [10]. Our study provides no insight into the effectiveness of vasopressin when used in conjunction with other vasopressors. These areas of study may provide more promise in future studies.

Although there are other untested mechanisms by which vasopressin might improve clinical outcome in verapamil poisoning, the results of our study do not support an improvement in MAP when used as a sole vasopressor agent. An increased death rate in the vasopressin group and a trend towards an initial decrease in CO may, in fact, point to a potential detrimental effect of vasopressin in the treatment of verapamil poisoning. Larger sample sizes would be needed to confirm these trends were not due to chance alone. The lack of efficacy of our experiment suggests that future study of vasopressin in the treatment of verapamil poisoning should concentrate on other areas with more promise of benefit.

\section{REFERENCES}

1. Ramoska, EA, HA Spiller, and A Myers, Calcium channel blocker toxicity. Ann Emerg Med, 1990; 19:649-53.

2. Stadlbauer, KH, HG Wagner-Berger, C Raedler, WG Voelckel, V Wenzel, AC Krismer, et al., Vasopressin, but not fluid resuscitation, enhances survival in a liver trauma model with uncontrolled and otherwise lethal hemorrhagic shock in pigs. Anesthesiology, 2003; 98:699-704.

3. Watson, WA, TL Litovitz, W Klein-Schwartz, GC Rodgers, Jr., J Youniss, N Reid, et al., 2003 annual report of the American Association of Poison Control Centers Toxic Exposure Surveillance System. Am J Emerg Med, 2004; 22:335-404.

4. Lee, D, Calcium Channel Blockers, in Toxicology Secrets, LC Ling, RF; et al., Editor. 2000, Hanlty \& Belfus Inc.: Philadelphia, PA. p. 80-82.

5. Brass, BJ, S Winchester-Penny, and BL Lipper, Massive verapamil overdose complicated by noncardiogenic pulmonary edema. Am J Emerg Med, 1996; 14:459-61.

6. Horowitz, BZ and KJ Rhee, Massive verapamil ingestion: a report of two cases and a review of the literature. Am J Emerg Med, 1989; 7:624-31.

7. Guidelines 2000 for Cardiopulmonary Resuscitation and Emergency Cardiovascular Care. Part 6: advanced cardiovascular life support: section 6: pharmacology II: agents to optimize cardiac output and blood pressure. The American Heart Association in collaboration with the International Liaison Committee on Resuscitation. Circulation, 2000; 102:I129-35.

8. Wenzel, V, KH Lindner, AC Krismer, EA Miller, WG Voelckel, and W Lingnau, Repeated administration of vasopressin but not epinephrine maintains coronary perfusion pressure after early and late administration during prolonged cardiopulmonary resuscitation in pigs. Circulation, 1999; 99:1379-84. 
9. Wenzel, V, KH Lindner, AW Prengel, C Maier, W Voelckel, KG Lurie, et al., Vasopressin improves vital organ blood flow after prolonged cardiac arrest with postcountershock pulseless electrical activity in pigs. Crit Care Med, 1999; 27:486-92.

10. Suzuki, Y, S Satoh, M Kimura, H Oyama, T Asano, $\mathrm{M}$ Shibuya, et al., Effects of vasopressin and oxytocin on canine cerebral circulation in vivo. J Neurosurg, 1992; 77:424-31.

11. Morris, DC, BE Dereczyk, M Grzybowski, GB Martin, EP Rivers, J Wortsman, et al., Vasopressin can increase coronary perfusion pressure during human cardiopulmonary resuscitation. Acad Emerg Med, 1997; 4:878-83.

12. Lindner, KH, B Dirks, HU Strohmenger, AW Prengel, IM Lindner, and KG Lurie, Randomised comparison of epinephrine and vasopressin in patients with out-of-hospital ventricular fibrillation. Lancet, 1997; 349:535-7.

13. Landry, DW, HR Levin, EM Gallant, RC Ashton, Jr., S Seo, D D'Alessandro, et al., Vasopressin deficiency contributes to the vasodilation of septic shock. Circulation, 1997; 95:1122-5.

14. Argenziano, M, AF Choudhri, MC Oz, EA Rose, CR Smith, and DW Landry, A prospective randomized trial of arginine vasopressin in the treatment of vasodilatory shock after left ventricular assist device placement. Circulation, 1997; 96:II-286-90.

15. Morales, D, J Madigan, S Cullinane, J Chen, M Heath, $\mathrm{M} \mathrm{Oz}$, et al., Reversal by vasopressin of intractable hypotension in the late phase of hemorrhagic shock. Circulation, 1999; 100:226-9.

16. Tsuneyoshi, I, H Yamada, Y Kakihana, M Nakamura, Y Nakano, and WA Boyle, 3rd, Hemodynamic and metabolic effects of low-dose vasopressin infusions in vasodilatory septic shock. Crit Care Med, 2001; 29:487-93.

17. Bartelstone, HJ and PA Nasmyth, Vasopressin Potentiation Of Catecholamine Actions In Dog, Rat, Cat, And Rat Aortic Strip.

Am J Physiol, 1965; 208:754-62.

18. Gaskill, HV, 3rd, KR Sirinek, and BA Levine, Hemodynamic effects of vasopressin. Can large doses be safely given? Arch Surg, 1983; 118:434-7.
19. Tanen, DA, AM Ruha, SC Curry, KA Graeme, and CG Reagan, Hypertonic sodium bicarbonate is effective in the acute management of verapamil toxicity in a swine model. Ann Emerg Med, 2000; 36:547-53.

20. Plewa, MC, TG Martin, JJ Menegazzi, DC Seaberg, and AB Wolfson, Hemodynamic effects of 3,4-diaminopyridine in a swine model of verapamil toxicity. Ann Emerg Med, 1994; 23:499-507.

21. Schwarz, B, P Mair, C Raedler, D Deckert, V Wenzel, and $\mathrm{KH}$ Lindner, Vasopressin improves survival in a pig model of hypothermic cardiopulmonary resuscitation. Crit Care Med, 2002; 30:1311-4.

22. Stadlbauer, KH, HG Wagner-Berger, V Wenzel, WG Voelckel, AC Krismer, G Klima, et al., Survival with full neurologic recovery after prolonged cardiopulmonary resuscitation with a combination of vasopressin and epinephrine in pigs. Anesth Analg, 2003; 96:1743-9, table of contents.

23. Malay, MB, JL Ashton, K Dahl, EB Savage, SA Burchell, RC Ashton, Jr., et al., Heterogeneity of the vasoconstrictor effect of vasopressin in septic shock. Crit Care Med, 2004; 32:1327-31.

24. Kern, KB, JH Heidenreich, TA Higdon, RA Berg, RW Hilwig, AB Sanders, et al., Effect of vasopressin on postresuscitation ventricular function: unknown consequences of the recent Guidelines 2000 for Cardiopulmonary Resuscitation and Emergency Cardiovascular Care. Crit Care Med, 2004; 32:S393-7.

25. Martikainen, TJ, JJ Tenhunen, A Uusaro, and E Ruokonen, The effects of vasopressin on systemic and splanchnic hemodynamics and metabolism in endotoxin shock. Anesth Analg, 2003; 97:1756-63.

26. Jackson, E, Vasopressin and the other agents affecting the renal conservation of water., in Goodman \& Gillman's: The Pharmacological basis of therapeutics, 10th Edition, LL Hardman JG, Editor. 2000, McGraw-Hill: New York. p. 789-808.

27. Sztajnkrycer, MD, GR Bond, SB Johnson, and AL Weaver, Use of vasopressin in a canine model of severe verapamil poisoning: a preliminary descriptive study. Acad Emerg Med, 2004; 11:1253-61. 